\title{
Hilbert arithmetic as a Pythagorean arithmetic: arithmetic as transcendental
}

\author{
Vasil Penchev, vasildinev@gmail.com \\ Bulgarian Academy of Sciences: Institute of Philosophy and Sociology: \\ Dept. of Philosophy of Science
}

Abstract. The paper considers a generalization of Peano arithmetic, Hilbert arithmetic as the basis of the world in a Pythagorean manner. Hilbert arithmetic unifies the foundations of mathematics (Peano arithmetic and set theory), foundations of physics (quantum mechanics and information), and philosophical transcendentalism (Husserl's phenomenology) into a formal theory and mathematical structure literally following Husserl's trace of "philosophy as a rigorous science". In the pathway to that objective, Hilbert arithmetic identifies by itself information related to finite sets and series and quantum information referring to infinite one as both appearing in three "hypostases": correspondingly, mathematical, physical and ontological, each of which is able to generate a relevant science and area of cognition. Scientific transcendentalism is a falsifiable counterpart of philosophical transcendentalism. The underlying concept of the totality can be interpreted accordingly also mathematically, as consistent completeness, and physically, as the universe defined not empirically or experimentally, but as that ultimate wholeness containing its externality into itself.

Keywords: eidetic, phenomenological and transcendental reductions; Hilbert arithmetic; Peano arithmetic; set theory and logic as Boolean algebra; qubit Hilbert space

\section{INTRODUCTION: THE IDEA OF HILBERT ARITHMETIC, OR ARITHMETIC AS TRANSCENDENTAL}

The paper imbeds the fundamental idea of Pythagoreanism that the basis of the being is arithmetical into a precisely defined mathematical structure, which can be also considered as a generalization of Peano arithmetic and thus, of arithmetic in Ancient Greece and meant by the Pythagoreans implicitly. Nonetheless, it is able to include as corresponding sub-structures propositional logic and set theory conserving consistent completeness once being interpreted as a first order logic including arithmetic and set theory simultaneously, and unlike Peano arithmetic due to the Gödel (1931) incompleteness theorems. Furthermore, Hilbert arithmetic is isomorphic or complementary to the qubit Hilbert space (respectively, to the separable complex Hilbert space of quantum mechanics) under a few additional conditions, and all wave functions (even belonging to both dual qubit Hilbert spaces) can be well-ordered as transfinite ordinal numbers, easily identifiable as transfinite natural numbers (i.e. the transfinite ordinal numbers less the least countable ordinal number). ${ }^{1}$

${ }^{1}$ Even more: it can be considered to be isomorphic to Minkowski space, in terms of which special relativity can be exhaustively reformulated, and further: to the Pseudo-Riemannian space of general relativity after curving Minkowski space, respectively the qubit Hilbert space and Hilbert arithmetic in the final analysis. However, even this is not all: the interpretation of Hilbert arithmetic allows for the phase space underlying thermodynamics (under either Boltzmann's, or Gibbs's, or also Einstein;s ontology) to be identified with a certain substructure contained in itself. 
However, the proper accent of the paper is the philosophical interpretation of Peano arithmetic therefore in a Pythagorean manner by means of Husserl's reduction interpreted once absolutely formally, and twice, according to the approach of transcendentalism as reinterpreted as followed in its essence by phenomenology (Penchev 2021 July 26). Practically, this means an ontological interpretation of information as classical as quantum, and justified by the origin of information from the totality (as to its ontological interpretation),

A preliminary notice is to articulate the exceptionally powerful, traditional prejudice claiming in default the ununifiable distinguishability of mathematics, physics, and philosophy, even confessing incommensurable concepts of truth ${ }^{2}$. The Gestalt of contemporary cognition (Foucault's "episteme") realized by the scientists in the three domains, and embodied in the legitimacy of their professional "guildies", does not allow for the eventual Great Unification (in physics) or the internal proof of consistent completeness (in mathematics) to step out beyond the exactly drawn proper boundaries, in the framework of which the "jurisdiction", i.e. cognizance and spirituality, of the science at issue are established and legitime.

As to philosophy always claiming generality by default, it is sharply distinguished from both physics and mathematics, not obeying either criterion of truth: philosophical truth is not and cannot be either physical (i.e. experimental) or mathematical (i.e. logically consistent truth). Thus, the "expressive postulate of scientific transcendentalism" being supported by a rigorous (even elementary) definition of the totality is necessary for philosophy to obey both physical and mathematical conception of truth, which in turn are merged between each other once adopted the postulate of the totality as relevant to each of them in a suitable form: correspondingly: the universe reinterpreted as the physical totality or the consistent completeness of mathematics as the same.

At first glance, the identification of the universe (an empirical and physical concept originating supposedly from experiments or sensual experience in the final analysis) and the consistent completeness (coming from logic and the tradition of mathematics, moreover grounded on the totality understood ostensibly (according to the objectioners) "scientifically" seems to be at least ridiculous. However, it relies on the same course of thought known as transcendentalism (starting from Kant's "Copernican revolution") and allowing for experience to be verified as both "phenomena" and "things by themselves" just in virtue of the postulate of the totality implying that verification.

For example and from the viewpoint of Platonism stating for all things by themselves to be "ideas" (including the mathematical concepts, statements and theories), they should be verified

So, the unification of the foundations of physics, mathematics, and philosophy seems to imply the particular unification only within physics and three of its most fundamental theories, namely: quantum mechanics (together with the Standard model); special and general relativity; and thermodynamics (independently of what the thermodynamic wholeness is interpreted to consists of: elements such as atoms, molecules, etc. (Boltzmann), states of wholeness (Gibbs), or macro-bodies interacting with their physical environment (Einstein).

${ }^{2}$ Accordingly: consistent completeness, experimental confirmation, number of "followers". The criterion of truth as to philosophy is shared by all art and humanities: that group of people granting for a certain doctrine to be true (or respectively, a piece of art to be a "masterpiece"). 
just as physical phenomena accessible by our experience and relevant experiments. That observation makes clear that the simultaneous implementation of Platonism and transcendentalism implies not only Pythagoreanism, but merges the former with "materialism" (e.g what Marx claimed to confess as opposite to Platonism, among which he enumerated also Hegel's doctrine). In other words, the distinction (and thus the opposition) of Platonism and "materialism" (not less, realism if it is understood as the necessary choice between them) makes sense only under the condition for transcendentalism to be rejected.

And vice versa: if transcendentalism is adopted (as here and even as scientific transcendentalism) the distinction of Platonism and materialism loses its meaning, and particularly, the empirical criterion of truth (as "adaequatio") and logical one (as "coherence") merges with each other just in virtue of the postulate of the totality: being as single as both adequate as coherent with itself. One can be convinced that both physics (namely, the theorems of the absence of hidden variables in quantum mechanics being consistent with its completeness) and mathematics (Gödel's dichotomy about the relation of arithmetic to set theory: either incompleteness or inconsistency) turn out to be at the doorstep of the totality as a scientific problem. However, quantum mechanics dare open the "door of the totality", and Gödel's investigation dare not, therefore and de facto returning or remaining out of the scope of transcedematlism and in the classical (yet Cartesian) dichotomy: either subject or object, tertium non datur.

The different behavior on the threshold of the totality is not difficult to be explained by the episteme of Modernity. Though science appeared in ancient Greece opposing the description of experience (e.g. Thucydides's history) and the deductive foundation from axioms and postulates (e.g. the "Elements" of Euclides about geometry), Galileo and Newton's idea of physical experiments searched for a relevant way to reconcile both approaches: physical theory as a mathematical description of experimental experience ${ }^{3}$.

Once modern physics has occupied that place in the episteme, mathematics has been forced to understand itself as a mathematical description at all, i.e. before or only independently of any empirical or experimental confirmation, being bodily and external to Descartes's "mind". This modern mathematics is constituted to be fundamentally incomplete, referring only to mental models created by formal rules, and opposed to the complete physics complemented by the bodily confirmation of all possible mental models.

Trying to justify itself only in its proper, i.e. only mental framework, the foundations of mathematics can be reduced to only two theories: arithmetic and set theory correlated to each other by the Gödel incompleteness: however now understood as a predetermined result for the episteme to which it belongs. Indeed, arithmetic can be interpreted as the model of mathematics

\footnotetext{
${ }^{3}$ The usual human experience is not "pure" enough to be able to distinguish sharply between alternative descriptions, and the concept of experiment as artificial as targeted to a certain distinction means: humans to "ask nature" by means of precisely formulated questions to avoid any ambiguity. That preciosity needs mathematics for the alternative descriptions to be different physical hypotheses experimentally distinguishable from each other.
} 
within itself, and set theory, as the model of complete physics including in its subject also the bodily world.

So, being a science obeying the modern episteme, mathematics finding itself on the threshold of the totality is forced to return backwards as far as that episteme, restricting it, is an unreflectable condition of its own possibility. On the contrary, referring to its legitime right to be epistemically complete, physics on the same "Rubicon" decides oppositely, to step out, on the boundary of the totality, until then dividing it from philosophy, by creating a complete physical theory, what quantum mechanics is after containing an internal proof of completeness: the theorems of the absence of hidden variables (Neuman 1932; Kochen, Specker 1967).

However, the epistemic analysis of the same theorems reveals immediately that they rely only on the separable complex Hilbert space, i.e a proper mathematical structure, and the completeness of quantum mechanics has been predetermined yet when it has adopted that space, both vector and functional and thus unitary, as its mathematical framework and fundamental model. This suggests that quantum mechanics has adopted (or better "appropriated" in virtue of its dominating place and recognized claims to be complete in the modern episteme) a properly mathematical proof based on a structure (namely, the separable complex Hilbert space) too distant from (and rather complicated to) arithmetic and set theory "canonized" as the foundations of mathematics.

The best decision is the episteme to be repaired. However this implies the direct intervention of philosophy into science to be able to accomplish amendments: a right of which philosophy has been depriving gradually more and more, century by century since the establishment of modern science therefore removing the option of fundamental epistemic changes forced its occurrence in the middle of the last millennium:

The "slogan" of the forthcoming paradigm revolution is Pythagorean, but rather synthesizing mathematics with physics and philosophy than mathematics to claim superiority over them. It needs only equality of rights with them: equality in the status of the mathematical "degree of reality". Mathematical reality is not only equally real to physical reality, but rather the same: and that identification of physical and mathematical reality is guaranteed by philosophy and its suggested intervention into science, investigated by the paper.

Scientific transcendentalism as a counterpart and identical dual twin of philosophical transcendentalism is what implies that approach, proclaimed here as interpretation of Husserl's call to philosophy to become a "rigorous science" and understood furthermore literally: as its unification with both physics and mathematics therefore sharing their formal and logical structure $^{4}$.

Once mathematics rejects its subordination as well as its secondary ontological place, it should take off the "Gödel glasses"of incompleteness and inferiority, only by which the episteme of Modernity allows for it to realize itself. "Removing the glasses" is absolutely sufficient to notice that quantum mechanics has appropriated its completeness, and the "triad" of

\footnotetext{
4 In fact, this pathos of "Logical investigations" (1900-1901) marked a turning point in Husserl's approach.
} 
propositional logic, set theory, and arithmetic in its foundations is isomorphic to the separable complex Hilbert space, moreover in a way able to medicate the Gödel "accusation" of arithmetic: either incomplete or inconsistent to set theory. That emancipation of mathematics rejecting obedience is the philosophical claim of Hilbert arithmetic.

Still one essential property of the intended new episteme is that information (strongly restricted to be not more than a specific scientific, and even only technical notion in the modern episteme) acquires a primary ontological status expessibly e.g. by the slogan: "All whether mental or bodily is information!"; even more: one can prove for information to be the ultimate substance of the world rather than only the next serial substance (after matter and energy), to which human cognition has managed to get. Speaking more precisely: one can prove that any possible meta-substance to the substance of information is isomorphic again to information.

That revolution of information depends crucially on the concept of quantum information allowing for quantum mechanics to be exhaustively reinterpreted as a theory of generalised information, namely quantum information and then understood as the information referring to infinite set and series unlike "classical" information related to finite ones (Penchev 2020 July $10)$.

Then (and by means of information both "classical" and quantum), the separable complex Hilbert space can demonstrate its symmetry absolutely, i.e. completely and consistently overcoming the hierarchy of level and metalevel, therefore reconciling them in idempotency. This approach is embedded in the dual structure of any Hilbert space (and thus, in that at issue). However, only the Standard model articulates it expressively to be able to underlie itself identifying the global space (i.e. that implied by the measuring apparatus of quantum mechanics) with the local space (i.e. that "inhabited" by quantum entities such as electrons, photoHs, etc.) as the same separable complex Hilbert space established still by quantum mechanics:

That identification in turn implies a fundamental bifurcation (or doubling) of the same space into the two identical copies of the local and global one: obviously, that bifurcation shares the formal structure of a bit of information demonstrated already in previous papers (Penchev 2021 April 12; 2020 October 20) as originating from the scientific concept of the totality. Then, the investigated quantum system can be recognized as isomorphic to the totality (defined scientifically) by the necessary mediation of information: the apparatus is that externality of the totality of the quantum system (especially emphasized by quantum holism) necessarily identified with the quantum system by itself in virtue of both identifications: that of the local and global spaces and that of the totality being in two identical hypostases in definition: externality and internality.

However, our prejudice (can be called "macroscopic chauvinism") prevents the identification of the local and global space (in fact a necessary condition for quantum mechanics and for the Standard model) to be accepted seriously (but only as a mathematical excess or curio) since the superiority of the macroscopic world over the quantum one according to the quantity of action or energy seems to people to be obvious and doubtless. On the contrary, still another "pair of glasses" must be taken off: those of "macroscopic chauvinism". In fact, the "size (of action and 
energy) does not matter" once quantum information is heralded to be the universal and ultimate substance of the world and the universe.

In other words, the ultimate universality of quantum information is due to the symmetry or identification of the global and local spaces as inherited from quantum mechanics and the Standard model as developed further: namely, as that of a bit information, embedded in advance into the relation of both dual Hilbert spaces as if "outside" (as far as embedding it "inside" would remain Hilbert space the same due to its infinite dimensionality). This means that the wave function (or respectively, the corresponding probability distribution to which it is its characteristic function) of an electron (e.g.) and that of my body (also for example), both considered as quantum systems, are indistinguishable from each other. The huge difference appears only after the interpretation of the different dimensions ("axes" of the separable complex Hilbert space or respectively, "qubits" of the qubit Hilbert space) as one or another type of quantum numbers, i.e. in the elementary and "external" choice of a single bit of information referring to whether the wave function at issue refers to the local space or to the global one:

However, after the wave function of any microscopic quantum entity needs an exact counterpart whether measured or measurable by the apparatus, this implies in turn the converse statement: any macroscopic entity involved in our usual experience (such as my body) possesses an exact "twin" being some microscopic quantum entity (e,g. such as an electron). The degree of how ridiculous (to common sense) the latter statement is can be visualised by the metaphor of "Schrödinger's cat": my body seeming to me to be only alive, in fact, is always both "alive-and-dead" simultaneously in virtue of the identification of its wave function with that of some microscopic entity (then, seen by the metaphor of "Schrödinger's cat" and reversely applied to "my body"). The paradox is seeming: not more than challenging the prejudice of common sense; however too harmful being shared by "scientific common sense" dominating in physics, therefore needing to be elucidated in detail:

My body is either alive (hopefully) or dead only in the past, but just both "alive-and-dead" in the present moment ${ }^{5}$. Indeed, the probability of a meteorite to fall onto my house (though extremely small) is a finite and non-zero one, therefore being able to exemplify the sense in which my body is both "alive-and dead" just now. Particularly, energy conservation needing the well-ordering of time according to the first Noether (1918) theorem can be relevant only to all the past moment of times, but not to the present moment when the well-ordering of time arrow has not managed to appear yet:

Thus, the interactions in the present moment as well as the processes of transforming the present moment into a corresponding past moment cannot obey energy conservation, but only quantum-information conservation generalizing the former, consequently conditioning the permanent violation of energy conservation (seeming to be a creation ex nihilo) due to the transformation of quantum information (due to entanglement) into energy (after decoherence).

\footnotetext{
${ }^{5}$ The length of the present moment can be defined as the period of the de Broglie wave associated with "my body" and much, much shorter than that of an electron (e.g), which allow for the idealization to be reduced to a point in the course of time, however only relatively (to an electron in the case). So, the same length is meaningless to be considered as a point to the much, much shorter length associable with the Milky Way (e.g.).
} 
However, the "scientific common sense of physics" represents the same phenomena mythologically, by the alleged "Big Bang" (the nonsense of which is shockingly obvious) as follows:

Energy conservation (eventually energy-momentum conservation in general relativity) is proclaimed incorrectly to be the most fundamental law of physics: therefore implicitly, but effectively postulating for the quantity of time to be identified as the quantity of the well-ordered past time, which is a necessary condition for energy conservation following from Emmy Noether's theorem (1918). In fact, this is a simplifying approximation under the condition that the length of the present moment (or the "zero point of here and now" in the space-time of relativity) is grantable to be zero and unlike the nonzero and finite value from it to any past moment of time. The additional assumption is necessary for the nature of time, which is meant by the length of the present moment, is reversible (due to the coherent state consisting of the superposition of all possible states of the investigated quantum system which ever it be) and just opposite to the irreversible well-ordered time of the past. That is: the simplification considers the present moment as an initial or final past moment therefore closing its way for research what happens on the "boundary" of the present and the past, moreover that boundary is a smooth area between two regions distinguishing fundamentally according to what kind of physical laws they obey; speaking loosely, "now" is quantum, and the past is classical:

If one adopts that temporal paraphrase of the usual postulate (formulated by Bohr) what quantum mechanics studies after quantum information and thus rejecting as energy conservation as that the "size (of energy) does matter", the system of both apparatus and quantum entity postulated fundamentally to be the investigated, one needs the invariance of the description as to "now" (for the quantum entity) and as to the past (for the apparatus). The same condition is trivially satisfied in classical mechanics for both kinds of time are identified rather implicitly and naively.

The real and gradual physical process of how "now" is transformed to become a past moment of time is neglected even in the "classical" quantum mechanics (and then, in the Standard model built on its basis) in virtue of the following circumstance. The invariance of "now" and the past implies the property of unitarity (indeed, embedded in the separable complex Hilbert space of quantum mechanics of the "classical" quantum mechanics), which implies particularly the "conservation of energy conservation in quantum mechanics" (Penchev 2020 October 5) since it allows "now" to be granted as the past equivalently.

However, this is only the "half of truth", at that, represented in a way closing the research of the other half: namely, the gradual process of how the coherent state of "now" can be transformed and is actually transformed in the past generating incredible huge energies and masses: just those "dark mass" and "dark energy" (totally, about 20 times more than the "visible" ones), the origin of which is rather tabooed by the dogmat of energy conservation than really mysterious.

In fact, the phenomena of the gradual transformation of "now" into the past are very wellknown, experimentally corroborated and studied in detail as those of entanglement and 
represented by the theory of quantum information as quantum mechanics could be exhaustively reformulated in the last decades of the 20th centuries.

Though all the "classical" quantum mechanics is a sub-domain of quantum information, this is furthermore a true sub-domain, the "jurisdiction" of which is definaole and restricted by energy conservation. On the contrary, the proper region and value of quantum information refers to its violation (where the "dark" origin of the matter and energy overcoming in the universe is "buried"), however obeying the more (most) general law of quantum information conservation implying particularly energy conservation under the additional condition for time to be "classical", i.e, the well-ordered time of the past.

The main property of entanglement (or respectively, that of the gradual decoherence of any coherent quantum state) consists in being out of time (and thus, out of space-time): even more, the time (space-time) appears as a result of it, namely, the state of absolute decorence (zero entanglement). Following the viewpoint of how the present moment is transformed into a past one, the way of this is by decoherence. In other words, time and space have not appeared in a distant time, the age of the universe after the Big Bang, but they come into being permanently (speaking figuratively, "each second": "figuratively", because any second is a result of the omnipresent decoherence).

If one integrates all decoherence all over the universe until the present moment, the usual picture of the expanding universe started from the mythical beginning of the Big Bang appears. That Big Bang itself violates all physical laws after the colossal universe can arise from nothing, ex nihilo. However, the question of how a similar nonsense can be the basis of physics is forbidden. Instead of that, the laws of physics neglecting the permanent and omnipresent decoherence are declared to be valid for both classical physics and "classical" quantum mechanics.

Alas, many experiments even legitimated by Nobel prizes ${ }^{6}$ demonstrated convincingly that the universe originating from the Big Bang remains about a 20 times greater part of all physics to be "dark" and fundamentally inaccessible: beyond the so made-up picture of the world. Though decoherence is out of space and time, it projects itself on the space-time "screen" (wrongly identified by contemporary physics as all claiming to be physical rather than only as a screen) as huge masses and energies (e.g. in accordance with Einstein's (1918) "Mach's principle"): properly, the notorious "dark matter" and "dark energy".

The relevant source of gravitation (as far as it satisfies the Einstein field equation of general relativity) is just the non-temporal process of decoherence, however represented on the temporal

${ }^{6}$ The Nobel Prize in Physics 2019 was awarded "for contributions to our understanding of the evolution of the universe and Earth's place in the cosmos" with one half to James Peebles "for theoretical discoveries in physical cosmology" (including for his research of dark matter)

https://www.nobelprize.org/prizes/physics/2019/summary/.

The Nobel Prize in Physics 2011 was divided, one half awarded to Saul Perlmutter, the other half jointly to Brian P. Schmidt and Adam G. Riess "for the discovery of the accelerating expansion of the Universe through observations of distant supernovae." https://www.nobelprize.org/prizes/physics/2011/summary/ 
screen of the past, space-time and completed decoherence. The same physical phenomenon (of how space-time appears) is seen from two gapped viewpoints (respectively, the past and the present) as ostensibly two absolutely disconnected physical phenomena: correspondingly, gravitation and entanglement. The former is described by general relativity, but the latter is yet seaking for the author of its theory in "Pirandello's manner" being neglected by the dominating Standard model and "classical" quantum mechanics (as far as both confess energy conservation rather than the generalizing quantum-information conservation).

So, if one takes off the "glasses of scientific common sense", only by which the visible on the "screen of space-time" can be identified as all makes sense to be physical (by the way, that taking-off is an action necessary for dark matter and dark energy to be investigated in detail), physics, mathematics and philosophy would not be already distinguishable from each other. The cherished theory of everything means first of all the unification of our cognition separable in those three fundamental branches if one has established for time to be only the time of the past, i.e. the well-ordered time relevant to the extreme of the completed absolute decoherence. Speaking figuratively, the study of entanglement as a huge, even crucially overcoming class of physical phenomena implies as a necessary condition the "entanglement" of physics, mathematics, and philosophy, too.

Consequently, transcendental arithmetic can be defined as those Pythagorean initial "elements" of arithmetic which merges with the foundations of physics and philosophy in a insetarble way, even as the same. Hilbert arithmetic generalizing Peano arithmetic can be identified as the mathematical viewpoint to that transcendental arithmetic.

\section{HUSSERL'S PHENOMENOLOGY AND SCIENTIFIC PHENOMENOLOGY: A SCIENTIFIC THEORY OF ONTOLOGICAL INFORMATION}

Hilbert arithmetic can be interpreted as a transcendental formal arithmetic by means of Husserl's phenomenology as far as he laid the foundations of a formal reinterpretation of transcendentalism in German philosophy following the paradigm of mathematics and especially logic to be rigorous non-experimental sciences and rather similar to each other. In other words, Hilbert arithmetic can be demonstrated as a properly mathematical structure able to underlie essential properties of Husserl's phenomenology (first of all, his conception of reductions: a rather innovative, formal and mathematical approach to philosophy, moreover permanently utilized by him to justify other notions and ideas in his doctrine). The same interpretation reveals information as a very special and ultimate concept supplying the smooth transfer between standard philosophical thesaurus and its rigorous and formal interpretation as Hilbert arithmetic, therefore verifying its implicit unifying basis in phenomenology ${ }^{7}$.

\footnotetext{
${ }^{7}$ During Husserl's lifetime, the theory and even the concept of information had not been existing, since both should be related to the middle of the last century and the fundamental papers of Claude Shannon and Norbert Wiener. Unlike the standard piety of history of philosophy directed to conserve philosophical doctrines literally and without any anachronic interpretations, Husserl himself reinterpreted the philosophical tradition following his own formal ideas originating from logic and mathematics. The present paper follows Husserl's interpretative approach to philosophy rather than that of history of philosophy, sacralizing and dogmatizing all past doctrines.
} 
A previous paper (Penchev 2021 July 26) suggested the way by which Husserl's phenomenology can be interpreted formally and informatically (i.e. by means of the theory of information as classical as quantum). The specific accent now will be the essential link between Hilbert arithmetic and the concept of information, and then and on this basis, the transcendental interpretation of Hilbert arithmetic in the traces of the cited article.

In fact, though introduced by the standard foundations of mathematics, namely arithmetic, propositional logic, and set theory in a way to contain them as substructures consistently, one may prove (or rather, to demonstrate elementarily) that Hilbert arithmetic is thoroughly an information structure consisting only of bits, qubits, and nothing else therefore as the qubit Hilbert space already involved by quantum mechanics as derivative from its fundamental formalism of the separable complex Hilbert space (Penchev 2020 August 25).

The approach for Hilbert arithmetic to be realized only and purely informatically consists in the consideration of (1) all bijections of "number-sake" elements, i.e. taking one with the same number from each of the three substructures: both twin Peano arithmetics, and the nonstandard interpretation of Peano arithmetic, on the one hand, and (2) each of those structures as a complete whole (i.e. as a set), on the other hand. The former, (1) represents an infinite series of bits sharing the unitarity of the qubit Hilbert space in turn originating from that of the separable complex Hilbert space, and the latter, (2): three "empty" qubits. Unifying both, one obtains the "philosophical" bit of information (as it was notated in the previous paper: Penchev 2020 July 2026). In other words, the formal and informational structure of Husserl's phenomenology as "quantum phenomenology" and the "informational reading" of Hilbert arithmetic (e.g. as just above) are the same rather than only isomorphic (this means that the discovery of any underlying structure is redundant).

However, the proper philosophical interpretation of the same bit consisting of three qubits turns out to be transcendental if one follows Husserl's phenomenology. This is almost obvious once the each of the three identical copies of a qubit are alleged to represent the "totality", and the meta-bit comprising all the three identical qubits, the definitive property of the totality formally and rigorously. In other words, the philosophical bit of information denotes the same "two times": ones extensionally, the totality as a qubit, twice intentionally, by the characteristic property of the totality as a bit of three qubits.

Just this option for Hilbert arithmetic to be interpreted transcendentally is what is meant by "Hilbert arithmetic as transcendental arithmetic". Thus, the idea of transcendental arithmetic related to the totality understood in a Pythagorean manner is not too far from its logical interpretation whether in a Hegelian or in a Husserian way. Though philosophy has moved away from mathematics still since Socrates's "humanitarian revolution" heralded human being (rather than e.g. the Pythagorean sacral "Number") to be the main subject of philosophy, the links between them have continued to exist during a few millennia.

The essential innovation without any precedent is the fundamental and ontological status of information as a quantity, but now absolutely emancipated from humankind's technique and 
technology as it appeared in the papers of Shannon (1948) or Wiener (1948) initially ${ }^{8}$ That primarily philosophical understanding of information is closer especially to Hegel's formal and ("dialectically") logical triad, but also to Peirce's triadic semiotics of sign (Nagl 2014) in a sense. Nonetheless remaining fundamental and ontological, it borrows from Shanon for information to be a quantity, but already, a Pythagorean kind of quantity, i.e. in the foundation of philosophy (in turn, allowing for their merging with those of mathematics and physics by transcendentalism whether philosophical or scientific).

Information becomes ontological in virtue of the "philosophical" bit of information immediately after its addition to Peano arithmetic "externally", at the next metalevel. Speaking in Pierce's manner (Marty 2014), arithmetic as a whole is understood semiotically, as the sign of the being, i.e, in a Pythagorean way in the final analysis. The addition of the "philosophical" bit of information just "outside" implies the involvation of the set of all natural numbers, on the one hand, and that of qubits and quantum information as "signs" of that kind of wholeness. Both result in Hilbert arithmetic as an inherently informational generalization of arithmetic, furthermore allowing for it to be interpreted as transcendental.

If human language is an invented and conventional sign of the being, arithmetic is that natural sign, or the sign by itself of the being, e.g. following Heidegger's way of defining Husserl's phenomenon in "Sein und Zeit" (1927). On the contrary, the human capability of speech, unlike any natural language, relies on arithmetic: a language shared by both being and nature $^{9} \ldots$ as well as by the computers ...

Finally, the particular identification of scientific and philosophical transcendentalism (distinguishing from each other in falsifiability) allowing for the theory of (quantum) information to be identified in turn with a philosophical doctrine of ontological information implies Husserl's phenomenology to be interpreted in that way and what the objective of the present paper is.

\section{HILBERT ARITHMETIC CONCISELY}

One can consider the "empty" qubit Hilbert space consisting of those empty qubits satisfies Peano axioms. The "empty qubit" can be introduced as the class of equivalence of all values which a qubit can possess. Thus,

\footnotetext{
${ }^{8}$ It had appeared in a fundamental and philosophical sense in Peirce's works, in his triadic model and semiotics. Anyway, Peirce's doctrine was not known enough in Europe at the beginning of the $20^{\text {th }}$ century, and first of all, it remains only qualitative, while the quantity of information (especially of quantum information) is crucial for any Pythagorean interpretation. One may object that Husserl's phenomenology is qualitative as well, however its links to logic, arithmetic and the foundations of mathematics are much closer while Peirce's semiotics is related rather to language and thus, to an ontology of language rather than to the ontology of logic and arithmetic.However, Husserl unlike Peirce did not utilize even the term of information initially seeming to be more applicable to language than to logic and arithmetics. Furthermore, Husserl did not demonstrate any affinity to Hegel's formal and triadic interpretation of transcendentalism, especially on the background of his relation to the original doctrines of Kant or Descartes.

${ }^{9}$ Nature can be distinguished from the being in the present context or in the trace of Heidegger's distinction of "ontic and ontological" as necessarily temporal.

10 "Empty" means to be granted as a free variable.
} 
Hilbert arithmetic consists of two dual Peano arithmetic(s) for the dual counterparts of the dual "empty qubit" Hilbert space. However, the direction of the function successor in each of them is to be opposite: " $\mathrm{n}+1$ " versus " $n-1$ ".

For the anti-isometry of the dual qubit Hilbert spaces (inherited from the corresponding anti-isometry of the separable complex Hilbert space) the two functions "successors" are "opposite" to each other. The twin arithmetic is to start from the "other end": respectively, from the " $\omega$ " defined as the least countable ordinal (thus, corresponding to a countable set):

" $\omega, \omega-1, \omega-2, \ldots \ldots 2+1,1+1,1$ ". The two twin arithmetics cannot be available simultaneously, or they are complementary to each other. To any natural number of one of them, just one transfinite "natural" (ordinal) number corresponds.

Inaccessible ordinals can be admitted also as rigorously less than " $\omega$ " (i.e. "transfinite inaccessible ordinal numbers") by the following construction. The inaccessible ordinals of first order are those, the set of all subset of which is a countable set; then, the inaccessible ordinals of second order are those all subset of which is a set corresponding to an inaccessible ordinal of first order; etc. From the viewpoint of Peano arithmetic, all the inaccessible ordinals (which can be restricted only to those of first order, due to additional considerations) are transfinite natural (ordinal) numbers. So, any natural number possesses just one transfinite and complementary counterpart belonging to the twin arithmetic as a standard natural number.

Hilbert arithmetic containing definitively inaccessible ordinals is out of the standard mathematics, the usual foundations of which are granted to be (Peano) arithmetic and set theory. A corollary: statements unprovable or false in standard mathematics can be provable (or even obvious) in Hilbert arithmetic (or in the framework of an eventual generalized, "Hilbert mathematics" justified by Hilbert arithmetic included in its foundations). Particularly, the Gödel incompleteness or Yablo's paradox are unprovable (i.e, they cannot exist) in Hilbert mathematics (Penchev 2020 August 25). On the contrary, Hilbert mathematics contains an internal proof of consistent completeness.

Three statements are valid: (1) Hilbert arithmetic is isomorphic to the qubit Hilbert space; (2) Hilbert arithmetic and the qubit Hilbert space are complementary to each other; (3) the previous two statements allows for the consistent completeness of quantum mechanics and Hilbert arithmetic to be interpreted as the same. The proof is based on the unambiguous mapping of all wave functions and all transfinite ordinal numbers after which the dual counterparts of both transfinite "halves" of the twin Peano arithmetics linked are isomorphic to the two dual qubit Hilbert spaces.

The usual interpretation of Peano arithmetic suggests that all natural numbers are well-ordered. Anyway, this property is based on the definition of the function successor (usually, " $n+1$ "). However, that definition can be changed in a way avoiding well-ordering, e.g. if the function successor be " $n=$ ", i.e. the series of natural numbers would be:

" $1,1=1,1=1=1,1=1=1=1$,...", (etc.) Obviously, it would finish cyclically: " $\omega=1$ ". That nonstandard interpretation possesses a cyclic structure decomposable into two 
complementary well-orderings; an illustration: the relation " = " can be decomposed into the two "complementary" relations: into " $\geq$ " and " $\leq$ ".

The usual understanding of "non-standard interpretation" is the construction of an enumerable (even finite) model in virtue of the Löwenheim - Skolem theorem (Arsenijević 2012). However, the opposite option is realized in the nonstandard interpretation of Peano arithmetic: a non-countable model of the countable Peano arithmetic is constructible ${ }^{11}$.

The axiom of choice is crucial in both cases. It generates an enumerable (i.e. countable) bijection, the sense of which can be realized as an enumerable model of a set of any arbitrary power as vice versa (the latter case is just the case at issue).

Then, why only one of the three copies of Peano arithmetic, distinguishable from each other by the way of the function successor to be defined, is granted as "nonstandard" needs additional explanations. The main reason is the symmetric structure of Boolean algebra, which can be ascribed to that Peano arithmetic alleged to be nonstandard, unlike the asymmetrical structure where addition and multiplication are defined arithmetically as usual, and "negation" and the second distributive law of Boolean algebra are not available in both rest twin copies of Peano arithmetic.

One needs a closer consideration of the interrelations of propositional logic and set theory. As it is well-known, both share isomorphically the structure of Boolean algebra (which is what underlies the Gödel 1930 completeness theorems), but set theory contains furthermore a rather metaphysical (though justified by relevant axioms rigorously) doctrine of infinity (the hierarchy of infinities), by the way, redundant for the foundations of mathematics, which is a special contribution of Georg Cantor ${ }^{12}$ and his unusual way of thinking directed to philosophical and even theological speculations (Steinhart 2009).

So, set theory, unlike any other mathematical theory as a first-order (in relation to propositional logic shared by all of them as the fundamental "zero-order" logic) is self-grounded in a sense (because of the shared Boolean algebra). Moreover, the axiom of choice allows for the additional Cantor doctrine of infinity to be represented equivalently by the complementary twin arithmetic. Then, the third, nonstandard interpretation of Peano arithmetic, comprising both standard twins, is to be equivalent to all sets or the set of all sets, however adopting Russell's (1902) antinomy not properly, but as a definitive property able to relate it to the totality (Penchev 2021 July 26).

Further, the relation of propositional logic, set theory, and arithmetic in the framework of Hilbert arithmetic is rather extraordinary to common sense: set theory (in the additional sense of

\footnotetext{
${ }^{11}$ It is non-countable in general because " $\omega-1$ " times repeated successively " $n=$ " can be replaced by any infinite ordinal for " $\omega$ " as well as in both standard twin Peano arithmetics: " $\omega-1$ " times repeated successively either " $n+1$ " or " $n-1$ ".

12 The proper philosophical (Oppy 2009) as well as philosophical, mathematical, and semantical (Sondheimer, Rogerson 2006; Usó-Doménech Selva, Requena 2016; Luis, Moreno, Waldegg 1991) sense of Cantor's conception of infinity can be demonstrated as underlying the greatest contemporary mathematical problems (Stewart 2013). The logical and mathematical contradiction consists in the relation of the axiom of induction in arithmetic, on the one hand, and the axiom of infinity in set theory, on the other hand (Keyser 1903; Baratella, Ferro 1993).
} 
Cantor's doctrine of infinity) as well as arithmetic are "non-convex" first-order logics in the following sense: both first-order logics can be considered as true subclasses of the zero-order logic of propositional logic (able to represent the "coherent state" of two "complementary" well-orderings opposite to each other).

One can notice that equality of all elements of the nonstandard Peano arithmetic "deforms" the arithmetical operations of "addition" and "multiplication" into the logical operations of "disjunction" and "conjunction" correspondingly.

There is a small problem with the exact correspondence of arithmetical "addition" and logical "disjunction" which can be overcome as follows. "Multiplication" can be considered directly as "conjunction", and the unambiguous mapping of namesake (or "number-sake") elements of the two twin Peano arithmetics can define the operation of logical "negation". The identification of those two operations is sufficient to imply the isomorphism of Boolean algebra. Furthermore. the "modulo two addition" can exemplify the logical inequality, which with either "negation" or "conjunction" defined arithmetically is enough for the identification of Boolean algebra.

So, the nonstandard interpretation of Peano arithmetic seems to be isomorphic to both propositional logic and set-theory: an observation needing to be discussed in detail and depth. Following the tradition of Western science, philosophy and culture, propositional logic and set theory are fundamentally distinguished as well as logic and mathematics. Since Cartesian dualism, they have been sometimes opposed even absolutely as a formal mental doctrine versus a formal physical (bodily) one (after the mathematization of physics in Modernity ${ }^{13}$ ).

However, Whitehead and Russell's "Principia mathematica" (any edition) suggested a fundamental unification of "proposition" $\backslash$ and "set" as the intensional and extensional aspects of the same, after which they are distinguishable only after Cantor's hierarchy of infinities, though partly reproduced in Russell's theory of types (Borkowski 1958). Anyway Cantor's theory of infinity as Russell's one can be considered in a "non-convex" way (as it is described above), so that the infinite hierarchy featuring both to be reduced to the corresponding identical idempotent repetitions between the two complementary counterparts of Peano arithmetic in Hilbert arithmetic and following the "lesson" of quantum mechanics by the separable complex Hilbert space translatable in mathematics by Bohr's concept of complementarity, to which the capability of quantum mechanics to be complete (internally provable) can be ascribed. Then, the structure of Boolean algebra is sufficient to embody that formal complementarity necessary for the consistent completeness of mathematics.

Any triple of "number-sake" elements of the nonstandard and both standard interpretations of Peano arithmetic constitutes a bit of information where the two complimentary standard elements are the two equally probable alternatives of a bit, and the nonstandard element represents a bit

\footnotetext{
${ }^{13}$ One can object meaning the late work of Husserl (1936), often notated briefly as "Die Krisis ..." that it blames the Galilean mathematization of physical qualities. Anyway, this would need a much more detailed consideration in relation to Hussel's earlier works and an estimation of his conceptual evolution.
} 
"before choice"14. If all elements of the qubit class of equivalence constituting an arithmetical unit are distinguished from each other, any unit would be transformed into a qubit.

Furthermore, the set of all natural numbers can be considered as a qubit since a qubit can be interpreted as a choice among the elements of an infinite set. So, the global and local structures coincide with each other and constitute the qubit Hilbert space.

The name "Hilbert arithmetic" is chosen following a few considerations: (1) Hilbert arithmetic is isomorphic to the qubit Hilbert space; (2) Hilbert arithmetic is able to justify Hilbert mathematics (unlike Gödel mathematics due to the Gödel incompleteness theorems: Penchev 2010) by an internal proof of consistent completeness. (3) Hilbert arithmetic embodies the Hilbert program of formalism ${ }^{15}$ though generalizing Peano arithmetic. (4) Hilbert arithmetic embodies the equivalence of the axiom of choice and the well-ordering "theorem" being a necessary condition of vector and function interpretations of Hilbert space identified by its conception.

\section{THE TRANSCENDENTAL MAPPING OF HILBERT ARITHMETIC INTO PEANO} ARITHMETIC

Scientific transcendentalism as mathematical transcendentalism implies a very extraordinary kind of bijection (which can be called "informational bijection"): "2:1" (respectively, "1:2"), each unit of which is a bit of information, and thus, any "informational bijection" defines a certain quantity of information (eventually infinite being a certain wave function).

Informational bijection is definitive for the totality, and for any other entity if one researches it as whole (eventually decomposable to elements or states as statistical thermodynamics does, for example). Any entity investigated by quantum mechanics is deprived from the option to be researched otherwise since it has embedded that informational bijection into the dual structure of its formalism of Hilbert space, and thus, any quantum entity turns out to be a "particular totality", or more precisely, a copy (whether a state or an element) of the totality.

However, the accent in this section will be only on the mathematical aspect of that new kind of bijection. The first question is whether, or under which additional conditions, its introduction can be consistent or consistent with the foundations of mathematics once it claims completeness, almost obviously. In other words, one should refuse the doubt that it is not a new edition of Hegel's "dialectic logic" inconsistent with formal logic, and even only to propositional logic.

Then, one, who is a mathematician, is to investigate whether informational bijection is consistent or can be consistent under additional circumstances with Boolean algebra. It is sufficient for informational bijection to be applied "outside", "externally" to Boolean algebra demonstrating that two Boolean algebras are equivalent to single one just as "two totalities" are equivalent to a single one (since the totality is single in definition). Indeed, if one considers

\footnotetext{
14 Thus, the unit of information, a bit, can be found in the relation of set theory (logic) and arithmetic: respectively, between infinity and finiteness or between proposition and calculation. The latter relation is well-known due to the model of calculation (as arithmetic as Boolean) by the Turing machine, the tape cell of which is equivalent to a bit of information.

${ }^{15}$ For example, following Webb's (1997) interpretation of Hilbert's formalism.
} 
(rather naturally), the eventual second exemplar of it as its negation, it will be identical for the absolute symmetries of "conjunction" and "disjunction" to "negation"("De Morgan's rules"), and thus their absolute identity rather than only idempotency.

Furthermore, one should prove that "external coincidence" of Boolean algebra with its negation does not contradict the law of noncontradiction in propositional logic valid inside it. Obviously, the case of its validity (being only "inside" provable) is unambiguously distinguishable from the case of its invalidity (being only "outside" provable). A contradiction would appear if both cases can be mixed under different circumstances. However this is prevented by the definition of the totality prohibiting it to be considered as a part of any alleged "meta-totality".

Anyway, whether Boolean algebra shares that general property of the totality is a statement needing a proof in turn, and it is not so elementary. Its stages are traced in detail in another paper (Penchev 2020 August 25) and can be summarized concisely as follows. Boolean algebra interpreted as the nonstandard interpretation of Peano arithmetic in the framework of Hilbert arithmetic can be identified (by virtue of the same) with a certain interpretation of the qubit (or respectively, separable complex Hilbert space), and after which it can share the proof of completeness of quantum mechanics (namely, the theorems of the absence of hidden variables). The interpretation of the qubit Hilbert space as Boolean algebra needs in a back reflection: (1) the class of equivalence of any possible values of a certain qubit as an "empty" qubit or as a unit of Hilbert arithmetic therefore generating two dual Peano arithmetics for each of both dual qubit Hilbert spaces; (2) the class of equivalence of both dual Peano arithmetics implying particularly the option for them to be identified as a single one (after the class of equivalence of the two isometric functions successors, i.e. identifying " $n+1$ " and " $n-1$ ", for example following the visualization of merging of two "weak" opposite well-orderings by the relations " $\leq$ " and " $\geq$ ' into "="). Since only two successive transformations, both into a class of equivalence, transform the qubit Hilbert space into Boolean algebra, the proved completeness of the former can be transferred immediately to the latter.

Speaking loosely, this can be called "the proof of the absence of hidden variables in Boolean algebra", a property postulated a long time ago after granting propositional logic as the zero-order logic able to underlie mathematics or correct thinking as well.

Then, the mapping meant in the title of the section (Hilbert arithmetic into Peano arithmetic) can be considered as a bijection (as well as the transcendental bijection) if Hilbert arithmetic is reduced to a well-ordered sequence of bits and any bit is mapped into its sequential number. This "projection" or "reduction" of Hilbert arithmetics is featured by two properties: (1) its qubits are "empty" to be able to be equated to the units of Peano arithmetic; (2) the property of unitarity of the qubit Hilbert space is abandoned.

The unitarity at issue underlies the "classical" quantum mechanics being embedded into the separable complex Hilbert space established to identify its two "versions": vector space (embodied in the initial matrix mechanics of Heisenberg) and functional space (in the initial 
ondulatory mechanics of Schrödinger) and implying the "conservation of energy conservation" in it (Penchev 2020 October 5).

If one has reduced the qubit Hilbert space to a series of bits as in (1) just above, the abandonment of unitarity means any bit to be reduced in turn to its two alternatives, which is the usual understanding of it, literally as a binary unit, the abbreviation of which is the term "bit" itself. If one pays enough attention, any bit interpreted as an elementary choice between two equally probable alternatives consists of two binary units, from which the one distinguishes the state before choice from that after choice, and the other one, the two alternatives of the choice properly. In other words, the omitted unitarity implies the bit as an elementary choice to be identified with a binary unit; also vice versa: the distinction of bit as a choice versus it as a binary unit (for example, as in the law of quantum teleportation: Penchev 2021 June 24) implies unitarity, and thus energy conservation as a direct corollary from quantum-information conservation (which is meant in the law of teleportation).

If untarity is abandoned and this is interpreted in terms of Hilbert arithmetic, the nonstandard interpretation of Peano arithmetic is what is missed. The mapping of Hilbert arithmetic into Peano arithmetic (which can be also granted as underlying Pythagoreanism) "brackets" both structures sharing Boolean algebra, propositional logic and set theory, therefore alleging Peano arithmetic to be absolutely sufficient as the foundation of mathematics (granted by the original Hilbert program unlike "Principia mathematica" inferring arithmetic from propositional logic, and then set theory, from both).

The claimed bracketing (only seemingly possible for the mapping of Hilbert arithmetic into Peano arithmetic) is not admissible in the present context for the following reason. It cancels the completeness of mathematics (which is due to Boolean algebra whether for propositional logic or set theory), once one tries to reduce it only to Peano arithmetic as its foundation and explicitly demonstrated as by Gödel (1930) completeness theorems as by his (1931) incompleteness theorems.

However, what arithmetics lacks in order to be complete is the "philosophical" bit of information (Penchev 2021 July 26). It can be granted to be added if Pythagoreanism is interpreted as an independent axiom able to complement Peano arithmetic in a way to reconcile it with set theory (implying the dual counterpart of Peano arithmetic as to the terms of Hilbert arithmetic) at the cost of including the world in mathematics ${ }^{16}$ unlike the standard "convex" worldview opposing them.

VI PEANO ARITHMETIC VERSUS LOGIC \& SET THEORY AND THE MEDIATION OF HILBERT ARITHMETIC: TRANSCENDENTAL TIME AND NON-TEMPORALITY

Peano arithmetic is often opposed to set theory due to the Gödel incompleteness theorem. However, the consideration of Peano arithmetic, naturally interpreted as a first-order logic and in the framework of Hilbert arithmetic, can be meant as isomorphic to set theory if one "brackets" the following two differences: (1) Peano arithmetic and set theory are idempotently

${ }^{16}$ This is meant if one introduces the definitively complete "Hilbert mathematics", as opposed to the standard viewpoint of "Gödel mathematics", correspondingly definitively incomplete (Penchev 2010). 
anti-isometric to each other; (2) Peano arithmetic is a "convex", i.e. external, additional structure to propositional logic, but set theory is "not-convex" to it as far as it contains within itself another copy of Boolean algebra identifiable as propositional logic together with the Cantor hierarchy of infinities in turn identifiable as another copy of Peano arithmetic ${ }^{17}$, though "nonconvex" to set theory.

Also vice versa: if both differences are meant, Peano arithmetic as a first order logic turns out to be complementary to set theory therefore, for example, describing the same (properly mathematics) only and purely arithmetically. Anyway, that arithmetical description of mathematics is incomplete definitively: it can be complete only complemented by its identical (neglecting those differences) counterpart of set theory.

Speaking figuratively, though identical like two "twins", the first-order logic of Peano arithmetic and set theory are anyway two individuals, and only both can represent exhaustively the complete "brotherhood" of them, from "whom" all their "descendants" (i.e. all theories in mathematics) originate. Following the same metaphor, Hilbert arithmetic is what is able to describe relevantly the "brotherhood of them": as their identity sharing the same Boolean algebra and Peano arithmetic as their distinction for the aforementioned two differences sufficient to be differed from each other, but only relatively: if both are available simultaneously.

Then, Hilbert arithmetic itself has a dual counterpart, "twin": the qubit Hilbert space equivalent to the separable complex Hilbert space of quantum mechanics. Both Peano arithmetics are replaced by their transfinite complements, representable as two dual Hilbert spaces consisting of wave functions well-ordered in virtue of the axiom of choice, just as the standard natural numbers of Peano arithmetic and following after them though the last natural number or the first transfinite number cannot be determined: they can exists only as a necessary corollary of the axiom of choice.

Then, the correspondence of the nonstandard Peano arithmetic (sharing isomorphically the structure of Boolean algebra) is still one (third) qubit Hilbert space for its fundamental property of unitarity: unlike the former two ones, it is not ordered in any way and thus, it is not well-ordered particularly. If taken into account that any qubit is interpretable as a certain quantum state as well as the distinction of bosons versus fermions (the latter share the principle of Pauli, consistent with their well-ordering, e.g. by the energy of those states), that "third edition" of the qubit Hilbert space corresponds to all bosons, and any pair of fermions sharing the same (energetic) state can be distributed: each of them in one of the two dual Hilbert spaces. The same duality of the qubit Hilbert space and Hilbert arithmetic (in a narrow sense) can be interpreted as the duality of the worlds of mathematics and physics (quantum mechanics) and implying a form of quantum Pythagoreanism, moreover merging into each other in their foundations.

\footnotetext{
${ }^{17}$ As far as Russell's theory of types (Borkowski 1958) is an analogue in logic of Cantor's hierarchy of infinities in set theory, it can demonstrate more visually the way of Peano arithmetic to be incorporated as the hierarchy of types where a certain natural number corresponds to any level.
} 
One can think of the eventual "supersymmetry" of bosons and fermions in terms of unitarity, which, in fact, seems to make them indistinguishable from each other, therefore generating trouble about their interpretation as two fundamental groups of quantum entities. ${ }^{18}$

A previous paper (Penchev 2021 April 12) discussed "transcendental time" in a rigorous and scientific sense following the idea of scientific transcendentalism. It was identified with the qubit (or separable complex) Hilbert space stating that it can be considered as a relevant generalization of the mathematical apparatus of classical physics investigating temporality by means of infinitesimal calculus invented by Leibniz and especially Newton right for physical theories, namely Newton's theory of gravitation or principles of physics,

Though involved by quantum mechanics for a specific area of cognition, one can advocate that the separable complex Hilbert space suggests a new and quite contemporary tool for researching temporality in a rigorous and mathematical method therefore applicable in many sciences even with quite different subjects, e.g. history, chemistry, cognitive science, etc. (along with physics, astronomy and cosmology) and appealing to a new, nonexistent science, "natural cybernetics of time" (Penchev 2021 February 25).

The fundamental and philosophical essence of the generalized approach to temporality consists in the assumption of quantum information, not only as a more general physical substance than energy, but as the ultimate and most general physical substance suggesting an internal proof of completeness. If temporality is linked to energy conservation and thus to the common worldview unrelain by the understanding of energy as the universal substance of the world (until now) and in accordance with Emmy Noether's (1918) "first" theorem, the conservation of quantum information (Penchev 2020 October 5) implies for nontemporal phenomena as physical as natural to be experimentally observable (rather than to exist "purely" and mathematically in virtue of the necessity due to logical consistency) just as an additional mass or energy after violating energy conservation in the framework of quantum-information conservation. "Dark matter" and "dark energy" are interpretable as those nontemporal violations (or projections on the usual physical temporality) after quantum information conservation (Penchev 2020 October 20). Another corollary is the rejection of the Big Bang as a real event: experimentally testable by astronomical objects older than the universe. The existence of those is inconsistent with the interpretation of the Big Bang as the real beginning of the universe (Penchev 2019).

\footnotetext{
${ }^{18}$ It consists in the problem: what makes a quantum entity either a boson or a fermion if one means their proper mathematical counterparts; or in other words, how one is to interpret the physical "spin" mathematically. Unitarity is linked to the physical sense (as Born's probability) only to the square of the modulus therefore allowing for two kinds of solutions: (1) of the same algebraic sign (bosons, and the absence of well-ordering; (2) of the opposite algebraic signs (fermions, and availability of well-ordering). Initarity unified them and thus implies (at least, af first glance) that supersymmetry at issue, which, however, is not observed in experiments. Nonetheless, that supersymmetry can be interpreted as the very well corroborated symmetry of matter and antimatter: the change of the charge sign is equivalent to the change of the spin between equivalent fermions and bosons after supersymmetry. Speaking loosely, one can say that unitarity forces supersymmetry to be observed as antimatter, therefore calling for a relevant explanation for the asymmetry of matter and antimatter. That is: why some quantum entities "prefer" to be bosons, and others, fermions.
} 
Summarizing the previous paragraphs in relation to physics, astronomy and cosmology, the tool for investigating both temporality and non-temporality by the qubit Hilbert space allows for the conditions and occurrence of temporality to be researched. The space-time "screen" being a metaphysical, unfalsifiable and necessary condition for physics and natural science until now can be discussed scientifically, i.e. in a falsifiable way after quantum-information conservation. As to other sciences such as history (Penchev 2021 April 6; 2020 October 18), chemistry (Penchev 2021 March 9; Penchev 2019), cognitive and neuroscience (Penchev 2021 February 25) where temporatility is not only essential, but crucially important and which could not take advantage of Newtopnian infinitesimal calculus created for physics and imposing conditions unsatisfiable by them, the new tool of the qubit Hilbert space allows for temporality pioneering mathematization just as Newton did to be utilized as a formal method.

As to mathematics, Hilbert arithmetic relevant (i.e as equivalent as complementary) to the qubit Hilbert space, but simultaneously being in the scope of its traditional subject of formal structures, allows for the solution of very difficult and heuristic problems (such as the seven CSI "Millennium problems") due to following circumstance of a rather philosophical issue. After many and many attempts undertaken by the most qualified among the most creative mathematicians of the planet - all failed! - one may admit, that those are unresolvable statements predicted by Gödel incompleteness theorems: so they are definitively unresolvable in contemporary mathematics until now. Or in other words, if they turn out to be resolved (e.g. as Poincare's conjecture by Perelman or Fermat's last theorem by Wiles), this has implicitly involved statements of mathematics based on Hilbert arithmetic, properly, for it is able not to admit unresolvable statements in a Gödelian sense and those absolutely correct "tricks" leading out of mathematics based on arithmetic and set theory can be proved (though not demonstrated ${ }^{19}$ ) explicitly (Penchev 20202 The relationship).

The previous paragraph contains a heuristic conjecture leading to another deductively: if mathematics based on Hilbert arithmetic (and practically, this is all contemporary mathematics since it reforms only the relations of propositional logic, arithmetics, and set theory to be consistent with each other and manages to interpret them as linkable to the foundation of quantum mechanics: the separable complex Hilbert space) is really both consistent and complete, all unresolvable statements in a Gödelian sense are resolvable in Hilbert mathematics (just that based on Hilbert arithmetic), and the former hypothesis is reasonable and justified, then all this implies that Hilbert mathematics is able to supply short and even elementary solutions of the most, most difficult mathematical problems nowadays, in fact originating rather from the room of mathematics in the cognitive episteme of Modernity than from real troubles in mathematics itself.

The opponents and objectors to Hilbert mathematics are mostly unreflecting, but following the tradition, according to which philosophy is rather harmful for its conceptions and proofs. On the contrary, the latter conjecture being a corollary from the former considers the great problems

${ }^{19}$ To be demonstrated explicitly as well is a question only of time and labor for any fundamental obstacles that are proved not to exist. 
of contemporary mathematics as needing a general, and thus, proper philosophical solution emancipating mathematics from its subordination to natural sciences studying reality itself unlike it and for this, ought to elaborate tools for them, which they choose and decide to utilize or not as well as how to use.

Hilbert mathematics is innovative, no less ontologically than properly mathematically, returning to a kind of Pythagoreanism (appeared yet at the dawn of both mathematics and philosophy in ancient Greece about twenty five centuries ago), relevant to the state of art in the new millennium. It claims that reality is no less mathematical than physical and thus emancipating itself from subordination; the unification is guaranteed by philosophy.

Seen from that viewpoint rather mathematically in a narrow sense, the relation of temporality and non-temporality (already admitted to be represented physically, e.g as "dark matter" and "dark energy") is just the relation of propositional logic and arithmetic in the context of set theory being articulated explicitly by Hilbert arithmetic, but only resurrecting ideas rather already embedded by Husserl in his phenomenology. Logic, and first of all, propositional logic as the zero-order logic both self-referential and self-identical definitively, can be that transcendental basis out of time able to support arithmetic (being well-ordered by the function successor and a mathematical counterpart of physical temporality), and then mathematics at all.

Anyway, Hilbert arithmetic, because of its inherent link to the unitarity of quantum mechanics, is able to add a few new meanings or connotations to the original Husserl approach. It connects propositional logic privileged to serve as a basis of mathematics and link to transcendentalism (interpreted here as scientific, particularly mathematical) with the equivalence of the axiom of choice and the well-ordering "theorem" in set theory. Furthermore, it considers the qubit Hilbert space as a generalized time which can be called "transcendental time" for its ability to unify temporality and non-temporality (the latter even interpreted metaphysically as "eternity").

\section{CONCISE CONCLUSIONS}

A consequence of the new, quantum Pythagoreanism and corresponding emancipation of mathematics consists in the proper philosophical sense of Hilbert arithmetic visible e.g. in the exhaustive formal and mathematical interpretation of Husserl's phenomenology being the subject of a previous paper (Penchev 2021 July 26). The present article following the same trace extends the approach to be rather more general, interpreting Hilbert arithmetic properly philosophically, namely transcendentally without restricting to Husserl's phenomenology or to a new, absolutely formal "quantum phenomenology".

The fundamental category of time as well as temporality being a necessary condition for natural science needs re-realization, but only the projections of that grandiose problem on the foundations of mathematics as Hilbert arithmetic ase considered here. Nonetheless, they correspond to essential changes in the foundations of physics and philosophy for the merging of their foundations, therefore, in the cognitive episteme of Modernity itself rather than only paradigms in each of those three sciences, absolutely separable from each other until now. 
Hilbert arithmetic, interpreted transcendentally, is a philosophical doctrine: however, it remains a rigorous and formal theory with a proper mathematical meaning and an experimentally verifiable basis as relevant to quantum mechanics. Being seen as a philosophical doctrine, it establishes an ultimate fundamental substance of all, whether physical or mental, therefore cancelling the main opposition underlying the cognitive episteme of Modernity (since Descartes).

\section{References:}

Arsenijević, M. (2012) "The Philosophical Impact of the Löwenheim-Skolem Theorem," in: M. Trobok, N. Miščević, B. Žarnić (eds.) Between Logic and Reality: Modeling Inference, Action and Understanding (Series: Logic, Epistemology, and the Unity of Science 25). Dordrecht: Springer, pp. 59-81.

Baratella, S., R. Ferro (1993) "A theory of sets with the negation of the axiom of infinity," Mathematical Logic Quarterly 39 (1): 338-352.

Borkowski, L. (1958) "Reduction of arithmetic to logic based on the theory of types without the axiom of infinity and the typical ambiguity of arithmetical constants," Studia Logica 8 (1): 283-295.

Gödel, K. (1930) "Die Vollständigkeit der Axiome des logischen Funktionen.kalldils," Monatshefte der Mathematik und Physik 37 (1): 349-360.

Gödel, K. (1931) "Über formal unentscheidbare Sätze der Principia Mathematica und verwandter Systeme I," Monatshefte für Mathematik 38 (1): 173-198.

Heidegger, M. (1927) Sein und Zeit. Tübingen: Niemeyer, 1984, p. 31.

Husserl, E (1900-1901) Logical investigations. Volume 1 \& 2, any edition.

Husserl, E. (1936) The Crisis of the European Sciences and Transcendental Phenomenology (any edition).

Keyser, C.J. (1903) "Concerning the axiom of infinity and mathematical induction," Bulletin of the American Mathematical Society 9 (8): 424-435.

Luis, E., A. Moreno, G. Waldegg (1991) "The conceptual evolution of actual mathematical infinity," Educational Studies in Mathematics 22 (3): 211-231.

Marty, R. (2014) "A Purely Mathematical Way for Peirce's Semiotics," in: T. Thellefsen, B. Sorensen (eds.) Charles Sanders Peirce in His Own Words. 100 Years of Semiotics, Communication and Cognition. (Series: Semiotics, Communication and Cognition). Berlin/ Boston: De Gruyter Mouton, pp. 415-420.

Nagl, L. (2014) "Peirce on Hegel, Pragmaticism, and "the Triadic Class of Philosophical Doctrines"," in: T. Thellefsen, B. Sorensen (eds.) Charles Sanders Peirce in His Own Words. 100 Years of Semiotics, Communication and Cognition. (Series: Semiotics, Communication and Cognition). Berlin/ Boston: De Gruyter Mouton, pp. 429-435.

Noether, E. (1918) "Invariante Variationsprobleme," Nachrichten von der Gesellschaft der Wissenschaften zu Göttingen. Mathematisch-Physikalische Klasse 1918: 235-257.

Oppy, G. (2009) Philosophical Perspectives on Infinity. Cambridge: University Press.

Penchev, V. (2021 July 26) "Quantum phenomenology as a "rigorous science": the triad of epoché and the symmetries of information," SSRN, https://dx.doi.org/10.2139/ssrn.3892039 or https://papers.ssrn.com/sol3/papers.cfm?abstract id=3892039, 
Penchev, V. (2021 June 24) "Two bits less" after quantum-information conservation and their interpretation as "distinguishability / indistinguishability" and "classical / quantum"," SSRN, https://papers.ssrn.com/sol3/papers.cfm?abstract id=3873123 or https://dx.doi.org/10.2139/ssrn.3873123

Penchev, V. (2021 April 12) "Both Classical \& Quantum Information; both Bit \& Qubit: Transcendental Time. Both Physical \& Transcendental Time," SSRN, https://papers.ssrn.com/sol3/papers.cfm?abstract id=3823665 or https://dx.doi.org/10.2139/ssrn.3823665

Penchev, V. (2021 April 6) "Modal History versus Counterfactual History: History as Intention," SSRN, https://papers.ssrn.com/sol3/papers.cfm?abstract id $=3818767$ or https://dx.doi.org/10.2139/ssrn.3818767.

Penchev, V. (2021 March 9) "The Generalization of the Periodic Table: The 'Periodic Table' of 'Dark Matter'," SSRN, https://papers.ssrn.com/sol3/papers.cfm?abstract id=3800823 or https://dx.doi.org/10.2139/ssrn.3800823.

Penchev, V. (2021 February 25) "Natural Cybernetics of Time, or about the Half of any Whole," SSRN, https://papers.Ssrn.com/sol3/papers.cfm?abstract id $=3750608$ or https://dx.doi.org/10.2139/ssrn.3750608 .

Penchev, V. (2020 October 20) "Two deductions: (1) from the totality to quantum information conservation; (2) from the latter to dark matter and dark energy," $S S R N$, https://papers.ssrn.com/sol3/papers.cfm?abstract id $=3683658$ or https://dx.doi.org/10.2139/ssrn.3683658 .

Penchev, V. (2020 October 18) "Natural Cybernetics and Mathematical History: The Principle of Least Choice in History," SSRN, https://papers.ssrn.com/sol3/papers.cfm?abstract id=3714119 or https://dx.doi.org/10.2139/ssrn.3714119.

Penchev, V. (2020 October 5) "Quantum-Information Conservation. The Problem About 'Hidden Variables', or the 'Conservation of Energy Conservation' in Quantum Mechanics: A Historical Lesson for Future Discoveries,” SSRN, https://dx.doi.org/10.2139/ssrn.3675319 or https://papers.ssrn.com/sol3/papers.cfm?abstract $\mathrm{id}=3675319$.

Penchev, V. (2020 August 25) "The Relationship of Arithmetic As Two Twin Peano Arithmetic(s) and Set Theory: A New Glance From the Theory of Information," SSRN, https://papers.ssrn.com/sol3/papers.cfm?abstract id=3656179 or https://dx.doi.org/10.2139/ssrn.3656179.

Penchev, V. (2020 July 10) "Quantum Information as the Information of Infinite Series," SSRN, https://papers.ssrn.com/sol3/papers.cfm?abstract id $=3630063$ or https://dx.doi.org/10.2139/ssrn.3630063,

Penchev, V. (2019) "From the Principle of Least Action to the Conservation of Quantum Information in Chemistry: Can One Generalize the Periodic Table?" Chemistry: Bulgarian Journal of Science Education 28 (4): 525-539.

Penchev, V. (2019) "Why Anything Rather Than Nothing? The Answer of Quantum Mechanics," in: I. Mladenov \& A. Feodorov (eds.), Non/Cognate Approaches: Relation \& Representation. Sofia: "Парадигма". pp. 151-172.

Penchev, V. (2010) "Unsolvability of the first incompleteness theorem; Gödel and Hilbert mathematics," Philosophical alternatives 19 (5) 104-119.

Shannon, C. E. (1948) “A Mathematical Theory of Communication," Bell System Technical Journal 27 (3) \& (4): 379-423 \& 623-666. 
Sondheimer, E. H., A. Rogerson (2006) Numbers and infinity: a historical account of mathematical concepts. Mineola (N.Y.): Dover.

Steinhart, E. C. (2009) “A Mathematical Model of Divine Infinity," Theology and Science 7 (3): 261-274.

Stewart, I. (2013) Visions of infinity: The great mathematical problems. New York: Basic Books.

Usó-Doménech, J.-L., J. A. N. Selva, M. B. Requena (2016) "Mathematical, Philosophical and Semantic Considerations on Infinity (I): General Concepts," Foundations of Science 21 (4): 615-630.

Webb, J.C. (1997) "Hilbert's formalism and arithmetization of metamathematics," Synthese 110 (1): $1-14$.

Whitehead, A. N., B. Russell (any edition) Principia mathematica.

Wiener, N. (1948) Cybernetics: Or Control and Communication in the Animal and the Machine. Cambridge (Mass.) MIT press. 